\title{
EVIDÊNCIAS PRELIMINARES DE ESTRATIFICAÇÃO VERTICAL DE POSTURA DE OVOS POR ALGUNS CULICIDAE (DIPTERA), EM FLORESTA NOMUNICIIPIO DE MANAUS - AMAZONAS *
}

\author{
José Lopes * * \\ Jorge R. Arias *** \\ J. Derek Charl Yood ****
}

\section{Resumo}

Foram instalados criadouros artificiais e $0 ; 1,0 ; 6,0$ e 11 metros de altura, numa floresta de terra firme em Manaus, Amazonas. O presente trabalho descreve um tipo de plataforma para estudo de estratificação vertical para postura de ovos de Culicidae e os resultados obtidos. Foram coletadas 10 espécies de Culicidae: Limatus durhami, Limatus flavisetosus, Culex bonnei, Culex mathesoni, Culex urichii, Culex originator, Trichoprosopon d. digitatum, Toxorhychites $h$. haemorrhoidalis, Wyeomyia aphobema e Orthopodomyia sp. As seis primeiras espécies foram coletadas em todos os níveis de alturas e suas populações diminuiram conforme a elevação. Tr. d. digitatum só foi encontrado até 6 metros de altura e sua população aumentou conforme a elevação do criadouro. To. $\mathbf{h}$ haemorrhoidalis foi coletado a 1 e 6 metros, Orthopodomyia sp. somente a 6 metros e $W$. aphobema somente a 11 metros de altura.

\section{INTRODUÇĀO}

Estudos de estratificação vertical ampliam o conhecimento ecoló- gico da população de insetos na floresta.

Diferentes espécies de insetos têm mostrado diferentes hábitos de estratificação nas florestas de terra firme nos arredores de Manaus (Penny \& Arias, 1981) e o conhecimento desses comportamentos é de fundamental importância para a interpretação da biologia das espécies. Muitos autores têm contribuído para o conhecimento de estratificação principalmente à altura de vôo de Culicidae. São mais restritos os dados de estratificação vertical sobre a criação desses Culicidae.

Entre insetos de importância médica os simulídeos têm sido bem estudados, no hábito de picadas em diferentes alturas (Ducke \& Beesley 1958; Fallis, 1964) ou estratificação de altura sobre as preferências de picada no corpo humano (Dalmat, 1955; Ducke \& Beesley, 1958; Fallis, 1964; Shelley et al., 1976). Mosquitos da família Culicidae tam-

\footnotetext{
* Parte da Tese de Mestrado do primeiro autor, apresentada à Universidade do Amazonas/Instituto Nacional de Pesquisas da Amazônia.

* Universidade Estadual de Londrina - Londrina - Pr.

*** Instituto Nacional de Pesquisas da Amazônia - Manaus - AM.

**** Intitute of Medical Reserarch - Papua New Guinea.
} 
bém foram estudados èm relação à altura de seu vôo ou de picada (Kruiff,. 1970; Gillies \& Wilkes, 1976; School et al., 1979), assim como em relação à altura de criação (Carpete et al., 1952 e Corbet, 1961).

Na região Amazônica, Shelley et al (1978), Lacey \& Charlwood (1980), estudaram a estratificação vertical em Simullidae. Também foi realizado trabalho de estratificação vertical com Lutzomyia por Shaw \& Laison (1968) em Belém, Pará, e Arias \& Freitas (1982) na região de Manaus.

Nesta região, os Dipteras $\mathrm{Ne}$ matoceras merecem atenção especial por parte da Saúde Pública, por exercerem ativo papel de veículadores de agentes patogênicos. São poucos os trabalhos de estratificação de postura, ou outros, que contribuam para um melhor conhecimento ecológico e possam servir de subsídios para medidas de controle sanitário destes insetos. Procurouse, em um curto período de coleta, evidenciar sinais de estratificação de postura de Culicidae, assim como descrever um novo tipo de plataforma para atender a esses objetivos.

\section{MATERIAL E MÉTODOS}

Os experimentos foram desempenhados numa floresta de "terra firme" com características de vegetação, solo e clima semelhantes à da reserva Ducke, descritas por Araújo (1967), Aubráville (1961), ou Penny \& Arias (1982). Está localizada no terreno do Campus Universitário da Fundação Universidade do Amazonas (FUA), na periferia da cidade de Manaus (030 08' 07" S. $60034^{\prime \prime}$ W.). Os experimentos foram montados próximos a uma das extremidades da mata, aproximadamente $200 \mathrm{~m}$ acima de um igarapé.

Para o estudo da estratificação vertical, foram construídas plataformas de madeira e instaladas em diferentes níveis de altura. $0 ; 1 ; 6 \mathrm{e}$ 11 metros. Estas plataformas tinham suportes para a fixação dos criadouros.Suas dimensões estão expressas na figura 1. A face inferior da plataforma tinha suportes para manter os criadouros em posição vertical. $\mathrm{Na}$ linha central da plataforma e a $14 \mathrm{~cm}$ das extremidades, dois ganchos foram parafusados na face superior e inferior. Uma corda foi utilizada para interligar as plataformas e como corda mestra para erguer as plataformas (fig. 2). Um barbante foi amarrado em pregos nos quatro cantos das plataformas e presos nas cordas para mantê-las em posição horizontal. Em um gaIho de árvore, a $13 \mathrm{~m}$ de altura, fixou-se uma carretilha e por esta passou-se a corda mestra para subir e baixar as plataformas.

Os criadouros artificiais utilizados eram fundos de frascos de álcool cortados em sua parte superior passando a possuir uma altura de $14 \mathrm{~cm}$, capacidade de $650 \mathrm{ml}$ (fig. 3).

Foram instaladas 4 plataformas, uma em cada altura, em 2 árvores, distantes 50 metros uma da 

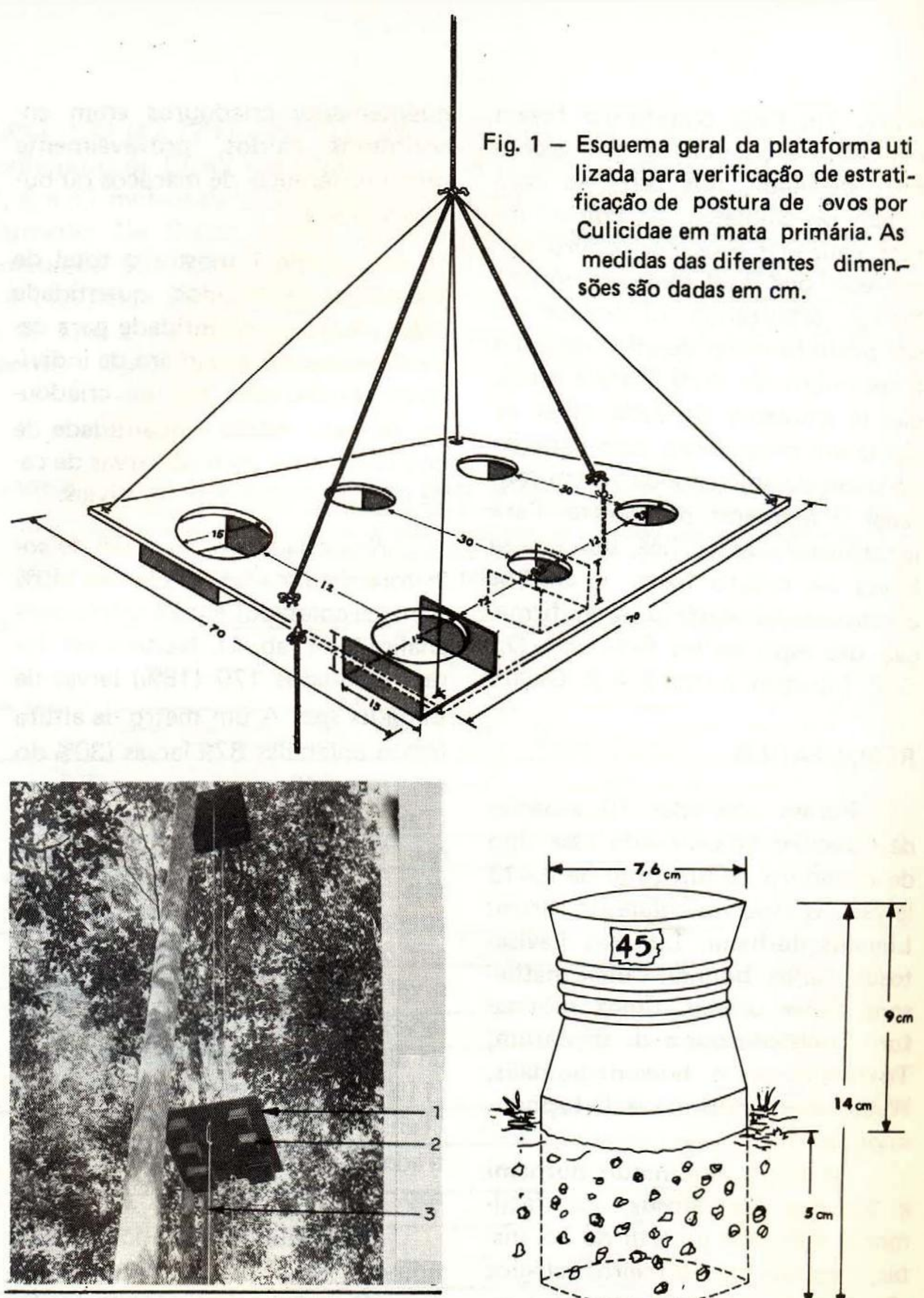

Fig. 2 -Vista geral das plataformas arma-

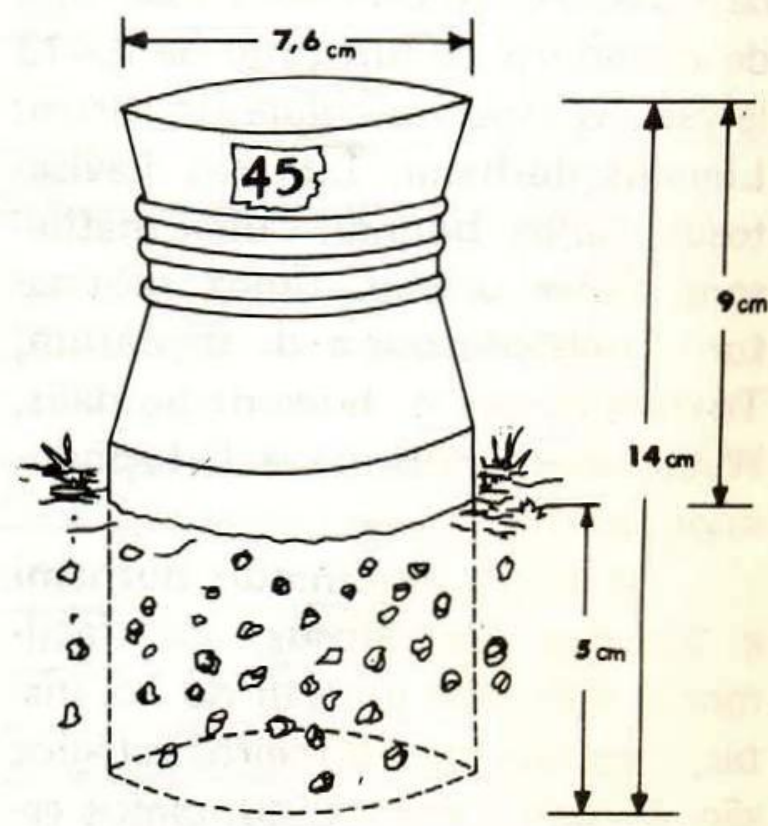
das na mata para estudo de estratificação de postura de ovos de Culicidae, onde se vêem:(1) plataforma, (2).suporte ventral, (3) corda de interligação das plataformas.

Fig. 3 - Esquema da garrafa plástica uti lizada como criadouro das larvas de Culicidae e suas dimensões. 
outra. Em cada plataforma foram colocados 4 criadouros, cheios com água destilada, nos orifícios com $9,3 \mathrm{~cm}$ de diâmetro. As coletas foram realizadas após o criadouro permanecer por duas semanas no ambiente, totalizando 12 coletas em um período compreendido de julho a dezembro de 1979 . Foram retiradas 6 amostras de cada nível de altura em cada árvore, com exceção do nível do solo; donde foram retiradas 3 amostras por coleta. Para identificação específica, utilizara-se larvas de quarto instar e adultos criados em laboratório. A confirmação das espécies foi feita pelo Dr. O. P. Forattini e Dra. E.A.B. Galati.

\section{RESULTADOS}

Foram coletadas 10 espécies de Culicidae colonizando esse tipo de criadouro de um total de 2.413 larvas. As espécies coletadas foram: Limatus durhami, Limatus flavisetosus, Culex bonnei, Culex mathesoni, Culex urichii, Culex originator, Trichoprosopon d. digitatum, Toxorhynhites $h$. hoemorrhoidalis, Wyeomyia aphobema e Ortopodomyia sp.

As larvas de Limatus durhami e Limatus flavisetosus, são facilmente separadas a partir do 3o. instar, mas, nos dois primeiros estágios são difíceis e por isso juntamos essas duas espécies aqui chamando-as de Limatus spp.

O número total de amostras analisadas (Tab. 1) não corresponde ao número programado, pois fre- qüentemente criadouros eram encontrados caídos, provavelmente por interferência de macacos ou outros animais.

A tabela 1 mostra o total de criadouros analisados, quantidade total de larvas, quantidade para cada espécie, maior número de indivíduos encontrados em um criadouro, número médio e quantidade de criadouro com zero de larvas de cada espécie, nos diferentes níveis.

Nos criadouros ao nível do solo foram coletadas 968 larvas (40\% do total coletado) em 26 criadouros analisados (Tab. 1). Neste nível, foram coletados $170(18 \%)$ larvas de Limatus spp. A um metro de altura foram coletadas 879 larvas $(30 \%$ do total) em 43 criadouros analisados (Tab. 1). A esta altura foram coletadas 147 (17\%) larvas de Limatus spp. A seis metros de altura foram coletadas 309 larvas ( $13 \%$ do total) em 56 criadouros (Tab. 1). Neste nível foram encontradas 71 (23\%) larvas de Limatus spp. A 11 metros de altura foram retiradas 257 larvas (11\% do total) em 64 criadouros (Tab. 1), neste nível foram encontradas 134 (52\%) larvas de Limatus spp.

Do número de criadouros e do número total de larvas coletadas, nos diferentes níveis de altura, foram capturados $22(2,27 \%), 17$ $(1,93 \%), 0(0,0 \%)$ e $3(1,17 \%)$ larvas de Culex bonnei, a $0 ; 1 ; 6$ e 11 metros respectivamente. Foram encontrados $598(60,74 \%), 555$ (63, 
14\%), $132(42,72 \%)$ e 92 (35,79\%) indivíduos de Culex mathesoni a 0 , 1, 6 e 11 metros de altura, respectivamente. De Culex urichii foram coletados $63(6,5 \%), 86(9,87 \%)$, $14(4,53 \%)$ e $3(1,17 \%)$ indivíduos a 0,$1 ; 6$ e 11 metros, respectivamente. Foram encontrados 85
$(8,78 \%), 34(3,87 \%), 15(4,85 \%)$ e $4(1,56 \%)$ indivíduos de Culex originator a $0,1,6$ e 11 metros, respectivamente, foram analisados 30 $(3,10 \%), 37(4,21 \%), 68$ (22,01\%) indivíduos de Trichoprosopon d. digitatum a 0,1 e 6 metros de altura respectivamente.

TABELA 1 - Mostra o número total de larvas coletadas, assim como o número total para cada nivel de altura - para cada espécle nesses niveis, número de criadouros analizados, - maior númèno en ontrado em um criadouro, o número médio de larvas por criadouro com zero larva, em experimento de estratificaçăo vertical en mata primária.

\begin{tabular}{|c|c|c|c|c|c|c|c|c|c|c|c|c|}
\hline \multirow{2}{*}{ ALIURA } & \multirow{2}{*}{ LAIDS } & \multicolumn{6}{|c|}{ E SPECIES } & & \multirow{2}{*}{\multicolumn{2}{|c|}{ 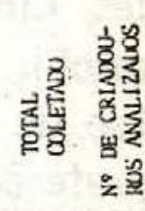 }} \\
\hline & & $\begin{array}{l}\text { Limatus } \\
\text { spp. }\end{array}$ & $\begin{array}{l}\text { Culex } \\
\text { botulei }\end{array}$ & $\begin{array}{l}\text { Culex } \\
\text { nathesoni }\end{array}$ & $\begin{array}{l}\text { Qulex } \\
\text { uricluii }\end{array}$ & $\begin{array}{l}\text { Culex } \\
\text { originator }\end{array}$ & $\begin{array}{l}\mathrm{Tr}, \mathrm{d} \text {. } \\
\text { digitatum }\end{array}$ & $\begin{array}{l}\text { To. h. } \\
\text { hacurrrrhoidalis }\end{array}$ & $\begin{array}{l}\text { Orthopomyia } \\
\text { sp. }\end{array}$ & W.aphobema & & \\
\hline \multirow{4}{*}{ 율 } & $N^{\circ}$ COLETAMO & 170 & 22 & 598 & 63 & 85 & 30 & - & - & - & \multirow[t]{4}{*}{968} & \\
\hline & $\begin{array}{l}\text { MIOR CON- } \\
\text { CENTRACAO }\end{array}$ & $4 \overline{3}$ & 22 & 150 & 20 & 71 & 10 & - & - & - & & \\
\hline & No MEDIO & 6,54 & 0,85 & 23,00 & 2,42 & 3,27 & 1,15 & - & - & - & & \\
\hline & $\begin{array}{l}\text { No DE CRIA- } \\
\text { DOUROS COM } \\
\text { ZERO LARVA }\end{array}$ & 10. & 25 & 3 & 18 & 24 & 20 & 26 & 26 & 26 & & 26 \\
\hline \multirow{4}{*}{ 番 } & $N^{\bullet}$ COLETADO & 147 & 17 & 555 & 86 & 34 & 37 & 3 & - & - & \multirow[t]{4}{*}{879} & \\
\hline & $\begin{array}{l}\text { MAIOR CON- } \\
\text { CENTRACAO }\end{array}$ & 43 & 12 & 56 & 37 & 17 & 15 & 1 & - & - & & 2 \\
\hline & Ne MEDIO & 3,41 & 0,39 & 12,91 & 2,00 & 0,79 & 0,86 & 0,07 & - & - & & \\
\hline & $\begin{array}{l}\text { NQ UE CRIA- } \\
\text { DOUROS ONM } \\
\text { 2ERO LNKVA }\end{array}$ & 24 & 41 & 10 & 37 & 38 & 38 & 40 & 43 & 43 & & 43 \\
\hline \multirow{4}{*}{ 送 } & NQ COLLTADO & 71 & - & 132 & 14 & 15 & 68 & 4 & 5 & - & \multirow[t]{4}{*}{309} & 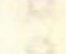 \\
\hline & $\begin{array}{l}\text { MAIOR CON- } \\
\text { CENTRACAOA }\end{array}$ & 16 & - & 25 & 6 & 15 & 27 & 1 & 3 & - & & as \\
\hline & Ne Nưlo & 1,28 & - & 2,36 & 0,25 & 0,28 & 1,25 & 0,07 & 0,09 & - & & $=$ \\
\hline & $\begin{array}{l}\text { NQ DE CRIA- } \\
\text { LOUROS COM } \\
\text { ZERO LARVA }\end{array}$ & 44 & 56 & 31 & 50 & 55 & 51 & 52 & 54 & 56 & & 56 \\
\hline \multirow{4}{*}{ 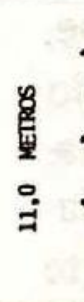 } & $N^{\bullet}$ COLETANO & 134 & 3 & 92 & 3 & 4 & - & - & - & 21 & \multirow[t]{4}{*}{257} & \\
\hline & $\begin{array}{l}\text { MUIOR CON- } \\
\text { CENTRAÇAO }\end{array}$ & 35 & 3 & 17 & 3 & 2 & - & - & - & 6 & & \\
\hline & NP NEDIO & 2.09 & 0,05 & 1,46 & 0,05 & 0,06 & - & - & - & 0,33 & & \\
\hline & $\begin{array}{l}\text { No DE CRIA- } \\
\text { DOUROS COM } \\
\text { ZERO LARVA }\end{array}$ & 45 & 63 & 42 & 63 & 62 & 64 & 64 & 64 & 59 & & 64 \\
\hline
\end{tabular}


Toxorhynchites h. haemorrhoidalis só foi coletado a 1 e 6 metros de altura. Esta espécie, foi encontrado um indivíduo em 3 criadouros a 1 metro e em 4 criadouros a 6 metros.

Orthopodomyia sp. só foi coletado em duas ocasiões a 6 metros de altura, num total de 5 larvas.

Wyeomya aphobema só foi coletado em cinco ocasiões a 11 metros de altura, num total de 21 larvas.

Para as espécies mais abundantes, Limatus spp., C. mathesoni e C. urichii, foi aplicado o teste de análise de variância para determinar a preferência de postura de ovos conforme aumenta a altura. As três espécies mostram preferência, diminuindo a densidade conforme aumenta a altura, sendo significativo

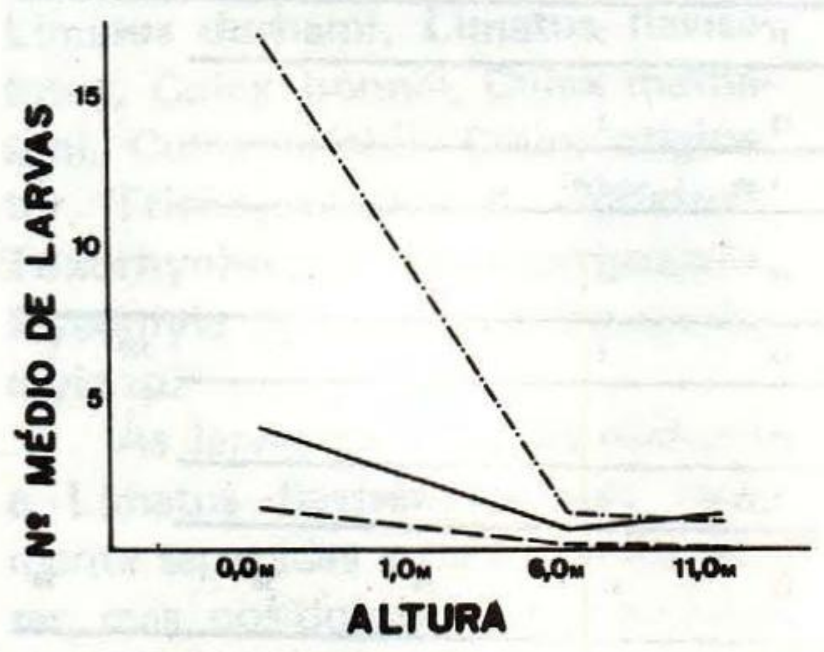

Fig. 4 - Variação populacional das larvas de Limatus spp. (-) , C. Mathesoni ( -.- ) e C. urichii (---), conforme se eleva o criadouro de zero a onze metros de altura. na ma ta alta. a nível de $0,1 \%(P>0,001)$ para todas as espécies testadas (Fig. 4). Ao nível do solo foi coletado um Coleoptero do gênero Copelatus, considerado pelo Dr. Splinger como espécie nova. Este Copelatus é predador das larvas de Culicidae.

\section{DISCUSSĀO E CONCLUSÃO}

Culex mathesoni e Limatus spp. são espécies que mais usaram este tipo de criadouro artificial para fazerem suas posturas de ovos em todos os níveis de altura. Suas populações caem conforme se elevam os criadouros, fato comprovado pelo teste de análise de variância, o que é significativo a nível de $0,1 \%$. A população de C. mathesoni cai uniformemente nos diferentes níveis, enquanto que Limatus mostra um aumento a 11 metros de altura. Esse aumento pode corresponder a dois eventos: este é o nível das copas, e alguns mosquitos vcarn orientados por um substrato, que, no caso, seria o solo e as copas das árvores, daí esse aumento populacional de Limatus a esse nível. A segunda hipótese relaciona-se à presença de Toxorhynchitesh $h$. haemorrhoidalis coletado somente a 1 e 6 metros. Esta última é uma espécie de Culicidae predadora (Lane, 1953; Forattini, 1968), influindo diretamente na população de Limatus, sendo que T. h. haemorrhoidalis coloca seus ovos isoladamente (Lane, 1953) o que explica as cole- 
tas em 7 ocasiões com uma única larva. A ausência desse predador a 11 metros de altura pode ter influenciado no aumento populacional de Limatus spp. Esses resultados são comparáveis com os encontrados para $\mathbf{T}$. brevipalpis coletado de 0 a 36,57 metros em uma floresta em Mpanga em criadouros artificiais de bambu (Corbet, 1961). Culex bonnei foi coletado ao nível do solo, a 1 e 11 metros. Sua população decai conforme aumenta a altura, desaparecendo a 6 metros, mas reaparecendo a 11 metros.

Culex urichii e C. originator foram coletados em todas as alturas, sua população diminuindo segundo a elevação do criadouro. Para a primeira, a análise de variância mostra esta flutuação significativa de $0,1 \%$.

Trichoprosopon d. digitatum só foi coletado até 6 metros de altura e sua população mostrou aumento segundo a altura.

Orthopodomyia sp. só foi coletado a 6 metros de altura. Esta é uma espécie que não colonizou os criadouros artificiais de plástico ao nível do solo e nem ao nível das copas. Assim, como é bem conhecido (Lane, 1953; Forattini, 1956) que muitos Culicidae procriam em bromélias que estão fixas nos troncos das árvores, é possível que estas sejam uma das colonizadoras dessas bromeliaceas.

Wyeomyia aphobema é a única espécie que só foi coletada ao nível das copas, aparentando ser uma espécie que faz suas posturas de ovos ao nível superior das florestas. Isto está de acordo com os resultados encontrados por Kruiff (1970) que coletou Wyeomyia acima dos níveis das copas.

Este experimento evidenciou a existência de uma estratificação em floresta de terra firme nas proximidades de Manaus.

A predação ao nível do solo é exercida pelo Copelatus sp. e acima deste pelo To. $h$. haemorrhoidalis. Não se liga a uma separação de nichos ecológicos, pois a larva do predador foi observada e coletada por nós, em criadouros naturais ao nível do solo, assim como, em uma oportunidade, em criadouro artificial de origem plástica, utilizados para outro experimento. $\mathrm{O}$ que poderia estar acontecendo, neste habitat, é a predação pelo Copelatus, ao nívèl do solo, das larvas de To. h. haemorrhoidalis. Como esta última deposita seus ovos isoladamente, a população é facilmente reduzida a zero. Isso foi comprovado em laboratório, quando colocamos juntos esses dois predadores. As larvas do 1o. e 2o. instar foram devoradas pelo Copelatus, imediatamente. As do 3o. e 4o. instar não foram predadas. Portanto, To. h. haemorrhoidalis é eliminado pelo Copelatus sp. logo no início de seu desenvolvimento, não sendo assim coletados por nós. Copelatus sp. não coloniza os criadouros acima do nível do solo, sendo aí a predação exercida pela larva de To. h. haemorrhoidalis. 


\section{SUMMARY}

Plastic artificial rearing cups were placed at 0,1,6 and 11 meters in a "terra firme" forest near Manaus, Amazonas. This work describes the types of platform used to study the vertical stratification of ovoposition of certain Culicidae. Limatus durhami, Limatus flavisetosus, Culex bonnei, Culex mathesoni, Culex urichi, Culex originator, Trichoprosopon d. digitatum, Toxorhynchites $h$. haemorrhoidalis, Wyemyia aphobema and Orthopodomyia sp. The first 6 species were collected at all levels their population size diminished with heigth. Only Tr. d. digitatum was found at 1 and 6 meters: its population increased with the heigth of the rearing pots. T. h. haemorrhoidalis was collected only at 1 and 6 meters. Orthopodomyia sp. was collected only at 6 meters and $\mathbf{W}$. aphobema only at 11 meters.

\section{REFERÊNCIAS BIBLIOGRÄFICAS}

ARIAS, J. R. \& FREITAS, R. A. 1982 - On the vectors of Cutaneous Leishmaniasis in the Central Amazon of Brazil. 3. Phlebotamine Sand fly stratification in a Terra Firme Florest. Acta Amazônica (no prelo).

AUBRÉVILLE, A.

1961 - Éude écologique des principalis formations végételis du Brésil; et contribution a la connaissance des forésts de L'Amazonie Brésiliense. França, Centre. Tecnique Forestier Tropical 268p.

ARAÚJO, U. C. de

1967 - A reserva florestal Ducke (Manaus); características e principais elementos florísticos e faun isticos protegidos. Atas do Simpósio sobre a Biota Amazônica, 7 (conservação da natureza e Recursos Naturais): 57-68
CARPETE, S. J.; GALINDO, P. \& TRÁPIDO, $\mathrm{H}$.

1951 - Forest mosquito studies in an endemic yellow fever area in Panama. Mosq. News, 12 (3)-156-164.

CORBET, P. S.

1963 - Mosquito breeding at different levels in and above the florest. Trans. Roy Entomol. Soc. Lon., 113(11) 249-358.

DALMAT, H. T.

1955 - The black flies (Diptera, Simuliidae) of Guatemala and their role as vectors of onchocerciasis. Smthsonian Institution, Washing ton, EEUU. v. 125 no. 1 425p.

DUCKE, B. O. L. \& BEESLEY, WN.

1958 - The vertical distribution of Simulium damnosun bites on the human body Ann. Trop. Med. Parasitol., 52. 247-281.

FALLIS, A. M.

1964 - Feeding and related behavior of female Simulüdae (Diptera). Parasitological Reviews, 15.439-470.

FORATTINI, O. P.

1962 - Entomologia Médica. São Paulo Universidade de São Paulo, v. 1. 662p.

1968 - Entomologia Médica. São Paulo, Universidade de São Paulo. v.2.506p.

GILLIES, M. T. \& WILKES, J. J.

1976 - The vertical distribution of some west African mosquitoes (Diptera-Culicidae) over open farmland in a

freswater area of the Gambia. Bull. Ent. Res, 66.515. 
KRUIFF, H. A. M. de

1970 - Aspectos of the ecology of mosquitos in relation to the transmission of arboviruses in Surinam, Leidem. 100 p. (Tese)

LACEY, L. A. \& CHARLWOOD, J. D.

1980 - On the biting behavior of some antropophilic Amazonian Simuliidae (Diptera).Bull. Entomol. Res.,70. 495-509.

LANE, J.

1953 - Neotropical Culicidae. São Paulo, Universidade de São Paulo, Indústria Gráfica Siqueira. v.2.

PENNY, N. D. \& ARIAS, J. R.

1981 - Insects of on Amazon Forest. Columbia University Press, New York. 2269p.

SCHOOL, P. J.; De FLOLIART, G. R. \& NEMENYI, P. B.

1979 - Vertical distribution of biting activity Aedes triseriatus Ann. Entomol. Soc. America, 72 (4) : 537539.
SHAW, J. J. \& LAISON, R.

1968 - Leishmaniasis in Brasil. II. Observations on enzootic rodent Leishmaniasis in the lower Amazon Region - The feeding habits of the vector Lutzomyia falviscutellata in reference to man, rodents and others animals. Trans. Roy. Soc. Trop. Med. Hyg., 62 : 396-405.

SHELLEY, A. J.; MELLO, J.A.S.N. de \& REES, R. G. O.

1976 - Observações sobre a transmissão de Oncocercose no rio Toototobi: Amazonas, Brasil. Acta Amazônica, 6 (3): 327-334.

(Aceito para publicação em 15/3/83). 